\title{
Association Between Body Mass Index with Cognitive Function and Activities of Daily Living In Elderly: A Cross-Sectional Study
}

\section{Samuel SE ${ }^{1}$, Senapathi $\mathrm{P}^{2}$, Shetty $\mathrm{S}^{* 3}$.}

${ }^{1}$ Professor \& Principal, Department of Community Medicine, A.J Institute of Medical Sciences \& Research Centre, Mangalore, Karnataka, India.

${ }^{2}$ Associate Professor, Department of Community Medicine, A.J Institute of Medical Sciences \& Research Centre, Mangalore, Karnataka, India.

*3 Post graduate student, Department of Physiotherapy, Laxmi Memorial College of Physiotherapy, Mangalore, Karnataka, India.

\section{ABSTRACT}

Background: Higher BMI has been often attributed with decline in cognitive function, mortality, and morbidity in elderly. However, few studies have reported high BMI as protective towards cognition and Activities of Daily Living (ADL). Accurate quantification of the role of $B M I$ in the incidence of cognition and performance is desirable in the face of the increasing prevalence of individuals with overweight and obesity and prolonged life span in the population. This study aimed to investigate the association of BMI with cognition and ADL in community living elderly.

Method: A cross sectional study was conducted among community living elderly. BMI and KATZ index scores were recorded from the respondents. Data were analyzed using SPSS version and a $p$ value of less than 0.05 was considered statistically significant.

Results: A total of 53 respondents enrolled in this study and was predominated by $(54.7 \%)$ males and (45.30\%) females. Karl Pearson correlation coefficient test found a positive correlation ( $r=0.33$ ) between BMI and Cognition and was found to be statistically significant $(p<0.05)$. Also, it was observed that moderately positive correlation exists between BMI and ADL $(r=0.64)$ and was found to be statistically very highly significant $(p<0.001)$

Conclusion: This study concluded that among elderly, higher BMI values were associated with better performance in daily activities and lower cognitive impairment.

KEY WORDS: Elderly, Body mass index, Activities of Daily Living, Cognition.

Address for correspondence: : Dr. Shetty S, PT., Post graduate student, Department of Physiotherapy, Laxmi Memorial College of Physiotherapy, Mangalore, Karnataka, India.

E-Mail: shettyshraddha89@gmail.com

Access this Article online

Quick Response code

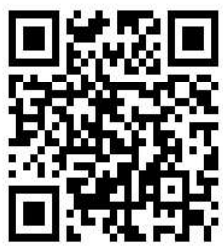

DOI: $10.16965 /$ ijpr.2021.163
Journal Information

International Journal of Physiotherapy and Research ISSN (E) 2321-1822 | ISSN (P) 2321-8975

https://www.ijmhr.org/ijpr.html

DOI-Prefix: https://dx.doi.org/10.16965/ijpr

Article Information

Received: 28 Jun 2021

Peer Review: 28 Jun 2021

Revised: 13 Jul 2021

\begin{tabular}{|ll|}
\hline \multicolumn{2}{|c|}{ Article Information } \\
\hline Received: 28 Jun 2021 & Accepted: 03 Aug 2021 \\
Peer Review: 28 Jun 2021 & Published (O): 11 Aug 2021 \\
Revised: 13 Jul 2021 & Published (P): 11 Aug 2021
\end{tabular}

$( c ) \longdiv { B r } + 4 0 - 8 \mathrm { A }$

\section{INTRODUCTION}

Depression and physical frailty as a dimension of quality of life increases the risk of cognitive impairment. At the biological level the effect of ageing on brain and cognition are widespread and have multiple etiologies.
Ageing has its effects on the molecules, cells, vasculature, gross morphology and cognition [1]. It must be underlined that health is not merely the absence of disease or infirmity, but a state of complete physical, mental and social wellbeing. In this framework, efforts have been made to explore possible factors 
influencing neuropsychological and physical performance in older age to prevent deterioration and promote good functioning [2].

Cognitive ageing, though a natural process, can interfere in the individual's functioning and quality of life [2,3]. Effective interventions have been proposed to prevent and optimize ageing related cognitive deterioration, considering protective factors such as cognitive training, physical activity, social engagement, and proper nutrition [4-6].

As physical and/or cognitive ability decreases in the elderly, care dependence arises to a point where an individual is no longer able to undertake the basic tasks that are necessary for daily life without the assistance of others [7]. The prevalence of dependence increases with age and is related to the presence of prior disease and fragility. Moreover, dependence relates to greater health and social resource utilization, more institutionalization, morbidity, and mortality, increasing overall health costs [8]. It must not be overlooked that dependence in the elderly is the main concern and cause of suffering and poor quality of life, and is significantly associated with suicide [9]. Despite dependence, an individual might well maintain his autonomy and retain the ability to make decisions and act in consequence on matters that affect him.

Apart from neuropsychological detriment, a disordered body composition is another expected significant ailment that comes along with age. Through the first 60 years of life weight gradually increases and thereafter it progressively decreases. Thus, even with a decrease in body weight, given a shortening of height, body mass index (BMI) is expected to increase between 1.5 and $2.5 \mathrm{~kg} / \mathrm{m} 2$ in both men and women [10].

Whereas some studies report that higher BMI in elderly is associated to a better daily life functioning [11], other studies have found that underweight or obese elderly subjects and those who had gained weight had more limitations on their daily living activities in comparison to those with a normal BMI and those who maintained their weight $[11,12]$.

In India the elderly account for $7 \%$ of the total population, of which two-thirds live in villages and nearly half of them in poor conditions. The elderly population in India increased from 20 million in 1951 to 57 million in 1991 and is expected to be 198 million in 206 and 326 million in $2050 ; 33 \%$ are living below the poverty line, $90 \%$ from the unrecognized with no social security [13]. Elderly people from the middle- and higher-income group are prone to develop obesity and its related complication due to sedentary lifestyle [14].

Although obesity may also affect cognitive function, the underlying mechanism to which it leads to cognitive deficits remains unclear. Evidence has shown that obesity affect cognitive functions in different ways for different people based on their age group. Prior studies have reported that obesity in later life was associated with lower risk of cognitive impairment, whereas obesity in midlife was associated with higher risk of cognitive impairment. This contradictory finding is known as obesity paradox caused by reverse causation [15].

Another explanation, excess body weight provides more energy savings and a stronger inflammatory response that could benefit to encounter acute illness. Some evidence for neuroimaging studies showed higher $\mathrm{BMI}$ in dementia patients with Alzheimer's disease (AD) was associated with greater volume of medial temporal cortex which indicates higher cognitive performance [16]. An additional explanation suggests that higher BMI in late-life individuals was associated with functional brain connectivity which served as a neuroprotection for cognition. Nevertheless, the exact mechanism by which obesity was less frequently associated with cognitive impairments remains unclear.

Although prior studies have associated BMI with cognition and daily performance of elderly, the results have been conflicting. As a result of the existing literature varies in association of BMI with cognition and activities of daily living, of note this association may vary depending on factors such as genetics, geographical areas, diet, culture and socio-economic reasons. Thus, this study aimed to evaluate association of BMI with 
cognition and activities of daily living in elderly residing in Southern India.

\section{MATERIALS AND METHODS}

A cross-sectional study was conducted among community living elderly referred by specialist for Physiotherapy, at selected tertiary care hospital in Southern India. Authorization and ethical approval were received from the Institutional Ethical Committee. Data was collected from 53 subjects ( $54 \%$ men and 45 $\%$ women) out of 60 subjects. The eligibility criteria were Men and Women aged 65 years and above, apparently healthy, and the ability to understand the local language was included. Severely impaired visual/ auditory acuity, immobilized elderly, neurodegenerative disorders, under psychotropic medications and living in palliative care or assisted care facility were excluded. All participants provided written consent on a locally translated informed consent forms.

Demographic data was collected including information about participants gender, previous occupation, age, education level, marital status, weight, and height. The World Health Organization (WHO) criteria for Asian population was used to classify the status of body composition. The participants BMI was calculated by dividing their weight in $\mathrm{kg}$ by their height in meters squared.

Participants were assessed for cognition using Montreal Cognitive Assessment Tool, where a score above 26 is normal. KATZ index of independence in ADL index tool (KATZ ADL) was used to screen for basic functional activities which consisted of six questions on physical functioning (bathing, dressing, toileting, feeding and continence) The possible score range of the ADL was 0-6 points, which was identified as dependent in at least one ADL (score 0-5) and independent in all ADL (score 6). Correlation of Cognition and Activities of daily living with BMI was estimated with Karl Pearson Coefficient test

Data was analyzed using SPSS 17.0 version for Windows and was depicted in number, percentage, correlation coefficient and $p$-value where less than 0.05 was considered statistically significant.

\section{RESULTS}

A total of 53 elderly were enrolled in this study. It was observed that the mean age of the study participants was $71.5 \pm 5.1$ years. The minimum age was 65 years and maximum age was 86 years observed in this study. Majority of the study participants were males (57.7\%) followed by (45.30\%) females. (Fig.1).

Among the whole participants, low BMI was associated with higher risk of cognitive impairment when compared to normal and higher BMI was correlated with a lower risk of cognitive impairment. (Table 1 \& Fig 2)

Based on ADL, KATZ index assessment scale, most of them were total independence $(62.2 \%)$ followed by (37.73\%) with moderate dependency.

Karl Pearson's correlation coefficient test found weak positive correlation ( $r=0.33$ ) between $\mathrm{BMI}$ and Cognition assessed through MoCA scale and was found to be statistically significant $(p<0.05)$. Also, it was observed that strong positive correlation exists between $\mathrm{BMI}$ and ADL ( $r=0.64)$ and was found to be statistically very highly significant $(p<0.001)$

\section{(Table 1 \& Fig 3)}

Table 1: Correlation of Body Mass Index with MoCA and ADL.

\begin{tabular}{lcc}
\hline \multicolumn{1}{c}{ BMI } & MoCA & ADL \\
\hline Pearson Correlation & 0.332 & 0.644 \\
Sig. (2-tailed) & 0.017 & 0 \\
$\mathbf{N}$ & 53 & 53 \\
\hline
\end{tabular}

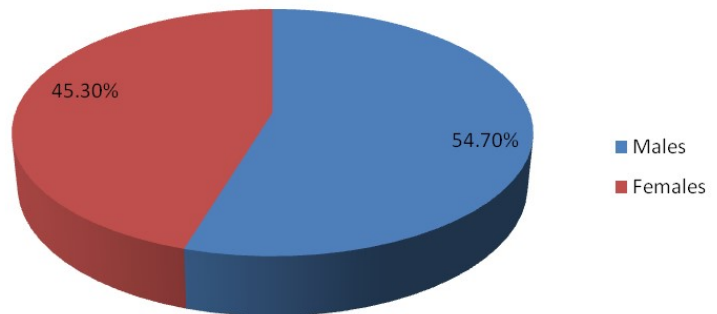

Fig. 1: Gender wise distribution of participants.

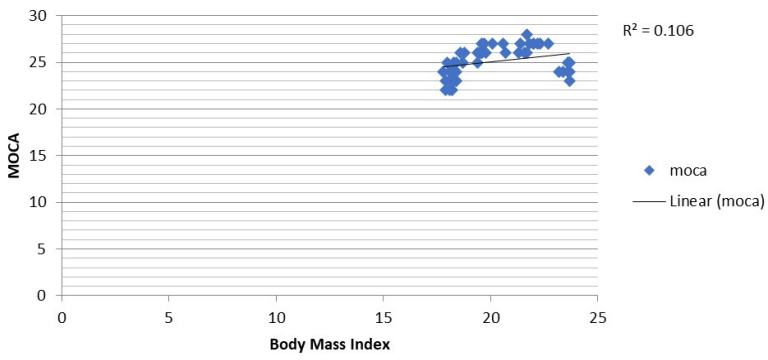

Fig. 2: Correlation of Body Mass Index with MOCA. 


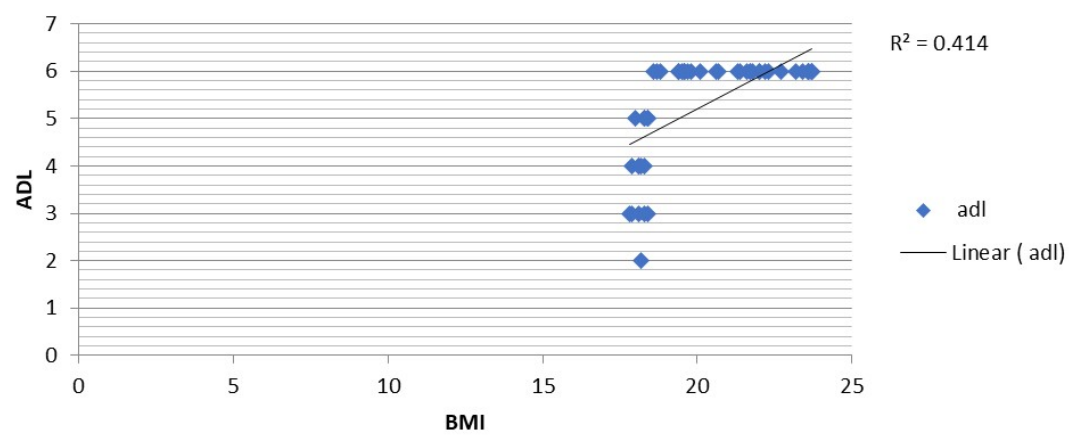

Fig. 3: Correlation of Body Mass Index with ADL.

\section{DISCUSSION}

Although prior studies have associated BMI with cognition, the results have been conflicting due to variability in geographical area, race, and other factors. Some studies showed high BMI in mid and late life is associated with a risk of developing cognitive impairment, but conversely the same is true for lower BMI measured in late life. However, the present study supports a clear association between $\mathrm{BMI}$ values and cognition.

In the present study, it demonstrated that cognitive impairment among elderly individuals in Southern India was affected by age and BMI. Elderly aged $65+$ were positively associated with cognitive impairment, with high BMI could be a strong factor to predict better cognitive function. The study also showed that elderly individuals aged 65+ were more likely to have cognitive impairment. This finding is consistent with previous study. After performing the analysis between age and $\mathrm{BMI}$, it was found that only higher BMI elderly individuals were significantly less likely to develop cognitive impairment. But, in contrast, two cohort studies from the western countries reported higher BMI with a higher risk of cognitive impairment, whereas the low BMI group did not have significant association $[17,18]$. This discrepancy may be due to different criteria used to classify BMI. In Asian population, obesity is defined as BMI greater than $25.0 \mathrm{~kg} /$ meter square according to WHO guidelines whereas in western countries it has been defined as more than $30 \mathrm{~kg} /$ meter square.

Our findings of lower BMI among the cognitive impaired supports the theory of obesity paradox reported by the few studies earlier [19]. Prospective studies so far from the American, Italian, and Korean elders have shown this association between lower $\mathrm{BMI}$ and less cognitive function or decline. A study on North Indian urban elderly reported a cognitive impairment prevalence rate of $8.8 \%$ [20]. In a comparable South Indian study on elderly aged 65 years and above, the prevalence was reported $11.5 \%$ [21]. Cognitive impairment in the present study was more among women compared with men. The difference in genders has been reported by several other studies earlier and attributed disadvantage among elderly women due to reduction in estrogen levels leading to greater cognitive decline than men [22]. However, the effect of our comparatively small sample size on the results cannot be ruled out. In late life it seems that being overweight and to a lesser extent obesity, is indicative of better health. The present study found that higher BMI related to better cognitive functioning.

Some previous studies suggest low BMI and some others suggest high BMI to be a risk factor for functional dependence $[23,24]$. Also, previous studies suggest that lower BMI is associated with better functional status [25]. Although in our study, we found a positive correlation between $\mathrm{BMI}$ and $\mathrm{ADL}$. This is supported by another study from Ozturk GZ et al.it shows overweight and obesity is protective against ADL. High waist circumference (WC) not associated with poor physical performance and decline in ADL in Lisko et al [26]. The combination of higher-level BMI and lower-level WC has been found to protect from the mortality in older adults; they also had a decreased likelihood of having decline in ADL. Another study found a similar correlation, LV YB et al. concluded that Higher BMI was associated with a lower risk of decline in ADL among Chinese adults aged 80 years and older [27]. 
Many studies have shown that sarcopenia, which is one of the main problems in elderly, is associated with the decline of muscle mass and strength and is a predictor of poor outcomes, including mortality, disability, and poor quality of life. Malunion and weight loss and the causes of sarcopenia which is associated with functional dependence in the elderly [28]. More attention should be paid on underweight, rather than the overweight or obesity for the prevention of decline in ADL after the age of 80 years. Accordingly, in our studies when BMI decreased, the ADL- performing ability decreased, possible because of the slowing down of the activity due to malnutrition or sarcopenia.

In the present study of 53 elderly people, 16 were categorized as underweight (30\%), 27 were normal (50\%) and 10 were of an overweight category (18\%). The classification of BMI was based on WHO criteria for the Asian population [29]. Although a decline in cognitive and physical functioning is common in older adults, our results suggest that underweight category are the most vulnerable group. Thus, prevention as well as intervention programs must respond to the needs of this markedly vulnerable group. Being a crosssectional study, no direction of the resulting association can be interfered. Underweight might be the cause or consequences of poor cognitive functioning and limited physical functioning, moreover all these factors might be influenced by other factors such as normal age-related deterioration and co-morbidities. Despite its limitations this study provided evidence of the association between disordered body composition, particularly an underweight $\mathrm{BMI}$, and poor cognitive and physical functioning. This association was independent of sex and age. Further research, particularly in a longitudinal fashion, shall expand this cross-sectional study by exploring the long-term effects and the vulnerability factors of the association that were found.

Several limitations of this study should be considered. Firstly, the sample size was small. Second, this study disregards the other factors that might have caused impairment in cognition and activities of daily living. This might have influenced the results.

\section{CONCLUSION}

This study concluded that among elderly, higher BMI values were associated with better performance in daily activities and lower cognitive impairment.

\section{Conflicts of interest: None}

\section{REFERENCES}

[1]. Peters R. Ageing and the brain. Postgrad Med J. 2006 Feb;82(964):84-8.

[2]. Lena A, Ashok K, Padma M, Kamath V, Kamath A. Health, and social problems of the elderly: a crosssectional study in Udupi taluk, Karnataka. Indian J Community Med. 2009 Apr;34(2):131-4.

[3]. Bharati DR, Pal R, Rekha R, Yamuna TV, Kar S, Radjou AN. Ageing in Puducherry, South India: An overview of morbidity profile. J Pharm Bioallied Sci. 2011 Oct;3(4):537-42.

[4]. You, H., Li, XI., Jing, Kz. Et al. Association between body mass index and health-related quality of life among Chinese elderly-evidence from a community-based study. BMC Public Health 2018;18:1174.

[5]. Momtaz YA, Haron SA, Hamid TA, Ibrahim R, Tanjani PT. Body Mass Index (BMI) and Cognitive Functions in Later Life. Curr Alzheimer Res. 2018;15(2):195200.

[6]. Hou Q, Guan Y, Yu W, Liu X, Wu L, Xiao M, Lü Y. Associations between obesity and cognitive impairment in the Chinese elderly: an observational study. Clin Interv Aging. 2019 Feb 15; 14:367-373.

[7]. Selvamani Y, Singh P. Socioeconomic patterns of underweight and its association with self-rated health, cognition, and quality of life among older adults in India. PLoS One. 2018 Mar 7;13(3):0193979.

[8]. Aune D, Sen A, Prasad M, Norat T, Janszky I, Tonstad $S$, Romundstad P, Vatten LJ. BMI and all-cause mortality: systematic review and non-linear dose-response meta-analysis of 230 cohort studies with 3.74 million deaths among 30.3 million participants. BMJ. 2016 May 4;353: i2156.

[9]. Schmeidler J, Mastrogiacomo CN, Beeri MS, Rosendorff C, Silverman JM. Distinct age-related associations for body mass index and cognition in cognitively healthy very old veterans. Int Psychogeriatr. 2019 Jun;31(6):895-899.

[10]. Horie NC, Serrao VT, Simon SS, Gascon MR, Dos Santos AX, Zambone MA, Del Bigio de Freitas MM, Cunha-Neto E, Marques EL, Halpern A, de Melo ME, Mancini MC, Cercato C. Cognitive Effects of Intentional Weight Loss in Elderly Obese Individuals with Mild Cognitive Impairment. J Clin Endocrinol Metab. 2016 Mar;101(3):1104-12.

[11]. Sund-Levander M, Wahren LK. The impact of ADL status, dementia, and body mass index on normal body temperature in elderly nursing home residents. Arch Gerontol Geriatr. 2002 SepOct;35(2):161-9. 
[12]. Bahat G, Tufan F, Saka B, Akin S, Ozkaya H, Yucel N, Erten $\mathrm{N}$, Karan MA. Which body mass index (BMI) is better in the elderly for functional status? Arch Gerontol Geriatr. 2012 Jan-Feb;54(1):78-81.

[13]. Tufan A, Bahat G, Ozkaya H, Tapcýoðlu D, Tufan F, Saka B, Akin S, Karan MA. Low skeletal muscle mass index is associated with function and nutritional status in residents in a Turkish nursing home. Gerontol Geriatr. 2016 Sep;19(3):182-186.

[14]. Quiñones AR, Markwardt S, Botoseneanu A. Diabetes-Multimorbidity Combinations and Disability Among Middle-aged and Older Adults. J Gen Intern Med. 2019 Jun;34(6):944-951.

[15]. Puzianowska-Kuznicka M, Kuryºwicz A, Walkiewicz D, Borkowska J, Owczarz M, OlszaneckaGlinianowicz M, Wieczorowska-Tobis K, Skalska A, Szybalska A, Mossakowska M. Obesity Paradox in Caucasian Seniors: Results of the PolSenior Study. J Nutr Health Aging. 2019;23(9):796-804.

[16]. Lisko I, Stenholm S, Raitanen J, Hurme M, Hervonen A, Jylhä M, Tiainen K. Association of Body Mass Index and Waist Circumference with Physical Functioning: The Vitality 90+ Study. J Gerontol A Biol Sci Med Sci. 2015 Jul;70(7):885-91.

[17]. Ozturk GZ, Egici MT, Bukhari MH, Toprak D. Association between body mass index and activities of daily living in homecare patients. Pak J Med Sci. 2017 Nov-Dec;33(6):1479-1484.

[18]. Sengupta P, Benjamin Al, Singh Y, Grover A. Prevalence, and correlates of cognitive impairment in a north Indian elderly population. . J Gerontol A Biol Sci Med Sci. 2014 Apr-Jun;3(2):135-143.

[19]. Yuenyongchaiwat K, Pongpanit K, Hanmanop S. Physical activity, and depression in older adults with and without cognitive impairment. Dement Neuropsychol. 2018 Jan-Mar;12(1):12-18.

[20]. Mac Giolla E, Kajonius PJ. Sex differences in personality are larger in gender equal countries: Replicating and extending a surprising finding. Int J Psychol. 2019 Dec;54(6):705-711.

[21]. Lv YB, Liu S, Yin ZX, Gao X, Kraus VB, Mao C, Yuan JQ, Zhang J, Luo JS, Chen HS, Zeng Y, Shi XM. Associations of Body Mass Index and Waist Circumference with 3-Year All-Cause Mortality Among the Oldest Old: Evidence from a Chinese CommunityBased Prospective Cohort Study. J Am Med Dir Assoc. 2018 Aug;19(8):672-678.e4.
[22]. Anstey KJ, Cherbuin N, Budge M, et al.Body mass index in midlife and late-life as a risk factor for dementia: A meta-analysis of prospective studies. Obes. 2011;Rev12(5):e426-e437.

[23]. García-Ptacek S, Faxén-Irving G, Cermáková P, et al.Body mass index in dementia. Eur. J. Clin.Nutr 2014;68(11):1204-1209.

[24]. Memel M, Bourassa K, Woolverton C, et al.Body mass and physical activity uniquely predict change in cognition for aging adults. Ann.Behav. Med. 2016;50(3):397-408.

[25]. Bruffaerts R, Demyttenaere K, Vilagut G, et al.The relation between body mass index, mental health, and functional disability: A European population perspective. Can. J. Psychiatry 2008;53(10):679688.

[26]. Bahat G, Tufan F, Saka B, et al.Which body mass index (BMI) is better in the elderly for functional status? Arch. Gerontol. Geriatr 2012;54(1):78-81.

[27]. Ford DW, Jensen GL, Still C, et al.The associations between diet quality, body mass index (BMI) and health and activity limitation index (HALex) in the Geisinger rural aging study (GRAS). J.Nutr. Health. Aging 2014;18(2):167-170.

[28]. Imai K, Gregg EW, Chen YJ, et al. The association of $\mathrm{BMI}$ with functional status and self-rated health in US adults. Gerontol. Geriatr. 2008;16(2):402-408.

[29]. Bahat G, Tufan A, Aydin Y, et al.The relationship of body mass index and the functional status of community-dwelling female older people admitting to a geriatric outpatient clinic. Aging. Clin. Exp. Res. 2015;27(3):303-308.

[30]. Misra, A Dhurandhar, N.V. Current formula for calculating body mass index is applicable to Asian populations. Nutr \& Diabetes 2019;9:3.
How to cite this article: Samuel SE, Senapathi P, Shetty S. Association
Between Body Mass Index with Cognitive Function and Activities of Daily
Living In Elderly: A Cross-Sectional Study. Int J Physiother Res
2021;9(4):3979-3984. DOI: $10.16965 /$ ijpr.2021.163 